\title{
XV International Materials Research Congress 2006 Held in Cancun \\ www.imrc2006.buap.mx
}

The XV International Materials Research Congress 2006 (IMRC 2006) and the V National Engineers NACE International Section Mexico Congress were held in Cancun, Mexico, at the Gran Meliá Cancún August 20-24, 2006. Pedro Hugo Hernández Tajeda (Benemérita Universidad Autónoma de Puebla, Mexico), president of the Mexican Materials Research Society, served as the general chair of the joint conference. In the welcoming address, he announced that over 1200 talks were scheduled, consisting of 174 invited talks, 415 oral presentations, and 657 posters from 40 countries.

The technical sessions included 22 symposia covering a range of topical areas, including nanostructured materials and nanotechnology, photovoltaics and solar energy materials, archaeology and art, ferro- and piezoelectricity, trends in polymer chemistry, building materials and technologies, biomaterials, ecomaterials, corrosion, and non-molecular solids. The conference also featured plenary lectures, tutorials, poster sessions and awards; roundtables on materials education and technological innovation; and an exhibit area for the display of equipment, instruments, products, software, publications, and services.

The conference also featured a special "Women in Science" networking luncheon. During the Luncheon, Karen Lozano of the University of Texas Pan American, USA and Noemi Elisabeth Walsöe de Reca, director of CINSO-CONICET-CITEFA, Argentina, were specially honored. Lozano and Walsöe de Reca talked about their difficult, though vastly different, paths en route to their success.

Support for IMRC 2006 was received from a number of institutions: the Secretaría de Educación Pública; the Vicerrectoría de Investigatión y Estudios de Posgrado; the Centro Universitario de Vinculación; Benemérita Universidad Autónoma de Puebla; the Instituto de Investigaciones en Materiales, UNAM; the Office of Naval Research International Field Office; JEOL; the Universidad Nacional Autónoma de México; the Centro de Investigación y de Estudions Avanzados, IPN; the Instituto Mexicano del Petróleo; the Office of Basic Energy Sciences; PONA, S.A. de C.V.; and the Instituto Potosino de Investigación Científica y Tecnológica (IPICyT).

Additional highlights of the events and research reported at IMRC 2006 are available online under "Materials Connections" on the Materials Research Society Web site at www.mrs.org.

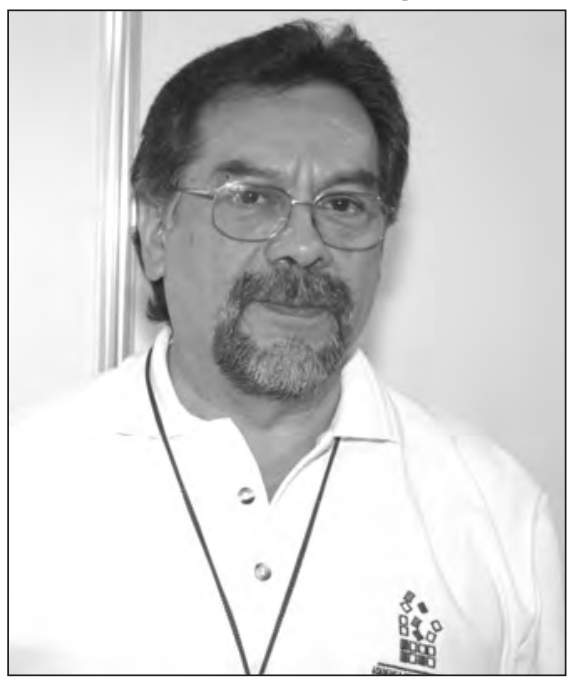

Pedro Hugo Hernández Tajeda (Benemérita Universidad Autónoma de Puebla, Mexico), president of the Mexican Materials Research Society, welcomed attendees to the XV International Materials Research Congress 2006 (IMRC 2006) and the V National Engineers NACE International Section Mexico Congress. The joint conference was held in Cancun, Mexico, at the Gran Meliá Cancún August 20-24, 2006.

\section{Plenary Sessions Address Energy, Catalysis, Nanomaterials, and Fractals}

In the first plenary lecture of the conference, entitled "Advanced Materials Innovations for New Technologies," Alex Ignatiev (Texas Center for Advanced Materials, Univ. of Houston, USA) took the audience on a wide-ranging tour of novel materials, with a focus on thin films and nanotechnology. This talk encompassed materials and processes from the probable to the fantastic and covered such fields as energy, superconductivity, environmental sensing, resistive computer memory, and materials at the physical-biological interface.

Beginning with a stark assessment of world electricity usage, which showed that two billion people on the planet have no access to electricity, Ignatiev described possible remedies in the form of advanced solar cells, fuel cells, and superconductivity. Replacing the $p-n$ junction of current solar cells with multiple-junction quantum wells results in enhanced absorption of light at the red end of the spectrum, increasing the efficiency of the cells. An
InGaAs triple junction has been made with 39\% efficiency. Multiple-junction quantum-well thermovoltaic cells extend this enhanced absorption into the infrared region, resulting in the direct conversion of heat into electricity, Ignatiev said.

Thin-film nanostructured fuel cells with an electrolyte layer only $1 \mu \mathrm{m}$ thick have been demonstrated to produce a high energy density of greater than $5 \mathrm{~W} / \mathrm{cm}^{3}$ while operating at $400^{\circ} \mathrm{C}$; currently available fuel cells operate at $900^{\circ} \mathrm{C}$. Ceramic superconductor technology using flexible $\mathrm{YBa}_{2} \mathrm{Cu}_{3} \mathrm{O}_{7-x}(\mathrm{YBCO})$ thin films is in the industrial prototype stage. This technology, which utilizes a thin layer of YBCO on a metal foil substrate, will probably replace the powder-in-tube method of producing wires from ceramic materials, Ignatiev said.

In the area of environmental sensing, Ignatiev described a thin-film nanostructured mid-IR laser with high power $(\sim 1 \mathrm{~W})$ that can operate at room temperature. Banks of 25 or more of these lasers with wavelengths in the range of $2-10 \mu \mathrm{m}$ could be packaged to form a small infrared spectrometer for the detection of biohazard compounds and other environmentally harmful chemicals.

A novel computer memory system called resistive random-access memory (RRAM) built with programmable nanoresistors could provide a new nonvolatile form of memory with tens of logic states, instead of the binary system currently used. Also in the realm of electronics, highenergy-density nanolayered capacitors made of thin films of BN could be used in extreme environments, including temperatures ranging from $-100^{\circ} \mathrm{C}$ to $800^{\circ} \mathrm{C}$.

At the physical-biological interface, Ignatiev detailed research being done on an optically active ceramic thin-film device that might be used as an artificial retina, restoring some level of sight to those with vision impairment. This ceramic device, which is photoresponsive at $600 \mathrm{~nm}$-in the green range of the spectrum- "could be used to replace rods and cones in the human eye," he said.

Ignatiev ended his plenary lecture with a flight of fancy, describing his vision of an automated lunar rover that would use regolith material-moon dust-to manufacture solar cells on the moon. Such solar cells could provide energy for a future lunar colony and possibly beam energy back to the Earth to reduce energy shortages here. The vacuum that is the moon's atmosphere suggests cleanroom conditions to Ignatiev, and the fact that regolith 
contains all the compounds needed to make solar cells suggests a good source of raw materials. He urged that "we take only the tools we need to make energy, not take the energy with us." Ignatiev and his colleagues have already succeeded in producing thin-film solar cells from regolith-like material in the laboratory.

Plenary speaker D. Yogi Goswami, codirector of the Clean Energy Research Center at the University of South Florida in Tampa, USA, said that energy use has been increasing steadily throughout the world at a rate of $2 \%$ per year ever since the dawn of the Industrial Revolution. Assuming this rate continues, world energy usage will double by 2037. In his lecture, entitled "Transitioning to a Renewable Energy Future," Goswami gave a sobering picture of the world's current reliance on fossil fuels and argued that renewable energy sources are the only hope for the near and distant future.

Goswami gave the following figures. Fossil fuels, including oil, coal, and natural gas, currently make up about $80 \%$ of the energy budget, with renewable resources representing $13 \%$ and nuclear power $6.5 \%$. The most optimistic predictions of oil reserves show a peak in production in 2030. Known reserves of uranium for nuclear power will be depleted by 2037. Coal may last for 80 years, but at a tremendous cost to the environment. At the current annual increase of $2.5 \%$ coal use in the world, $\mathrm{CO}_{2}$ emissions linked to global warming will increase from 300 gigatons in the atmosphere today to 8000 gigatons by 2030 .

The German Advisory Council on Global Changes estimates that renewable energy sources such as solar power, wind, biomass, and hydropower must be able to meet $50 \%$ of the world's energy needs by 2050 in order to sustain living standards. Fortunately, Goswami said, renewable energy sources will meet this challenge if research and development are accelerated, starting now. Already, new materials and turbine technologies are causing energy from wind power to double about every two years. Biomass, including biodiesel fuels and ethanol, accounts for $11 \%$ of the world's energy today, but Goswami said this is only $18 \%$ of its current potential. The increased efficiency in solar photovoltaic cells and their incorporation into aesthetically pleasing architectural designs is reducing the cost of solar-generated energy. In addition, power plants based on solar thermal electric power technologies are generating electricity at a cost of only $\$ 0.11 / \mathrm{kW} \mathrm{h}$. Based on these success stories in the renewable energy field,

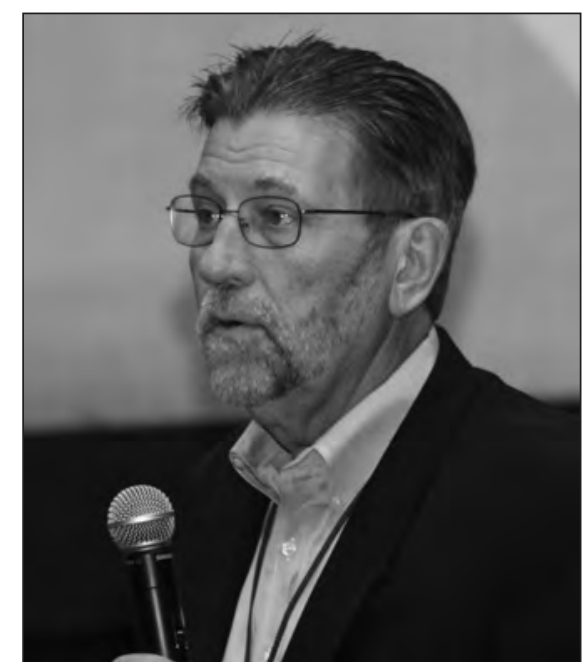

Plenary speaker Alex Ignatiev (Texas Center for Advanced Materials, University of Houston) talked about materials and processes from the probable to the fantastic.

Goswami concluded that "only renewable energy sources will provide for our energy future."

Scanning electrochemical microscopy (SECM) is a new technique for the rapid screening of potential bimetallic and trimetallic catalysts for the reduction of oxygen in fuel cells. Allen J. Bard, an electrochemist from the University of Texas at Austin, USA, explained the technique and demonstrated its usefulness in his plenary lecture, "The Application of Scanning Electrochemical Microscopy in Materials Chemistry-New Approaches in Electrocatalyst Discovery."

SECM utilizes an ultra-microelectrode whose tip diameter ranges from $10 \mu \mathrm{m}$ to $20 \mu \mathrm{m}$. By measuring current at the tip as it is scanned over an array of potential catalysts, researchers can rapidly screen large numbers of compositions for electrocatalytic activity. A "hit" occurs when current is generated at the tip as it passes over a sample of a particular composition; if no current is generated, the composition is rejected. The technique essentially performs surface electrochemistry with a combinatorial chemistry approach.

The platinum used as an electrocatalyst in fuel cells now is "too expensive and too rare," Bard said. To find a replacement, he and his colleagues follow a six-step approach: (1) try bimetallic and trimetallic compositions; (2) screen the compositions rapidly using SECM; (3) optimize the synthesis of any promising materials; (4) use the optimized catalyst in an actual fuel cell;
(5) characterize the catalyst using x-ray diffraction and other techniques; and (6) carry out detailed theory analysis on promising materials. The theory developed in step 6 is then used to inform the search in the next round of screening. While he admits that this process is backwards from that followed by many researchers who develop a theory first, Bard contended that "it only pays to make detailed theoretical studies when you have good materials around."

In the reduction of oxygen, the first step is the breaking of the $\mathrm{O}-\mathrm{O}$ bond. Elements that are good at oxygen bond breaking, such as Co, tend to produce a low electrochemical potential, while noble metals that produce a good electrochemical potential are ineffective at bond breaking. By combining elements like $\mathrm{Co}$ and $\mathrm{Pd}$, or like $\mathrm{Pd}$, Co, and Mo, into a single catalyst, the researchers hoped that the strengths of both types of elements would combine to produce an effective catalyst.

Experimentally, Bard and his colleagues prepared arrays of mixtures of metals on a carbon substrate using automated dispensing tips. The result was an array containing, for example, compositions of $\mathrm{Pd}$ and Co ranging from $100 \% \mathrm{Pd}$ to $100 \% \mathrm{Co}$, with intermediate compositions of $90 \%$ $\mathrm{Pd}-10 \% \mathrm{Co}, 80 \% \mathrm{Pd}-20 \% \mathrm{Co}$, and so on. SECM analysis showed that the $80 \%$ Pd-20\% Co mixture was a better electrocatalyst than pure $\mathrm{Pd}$, although it still lagged behind the Pt standard.

Analysis by scanning electron microscopy and x-ray photoelectron spectroscopy revealed the following mechanism: $\mathrm{O}_{2}$ binds to a Co site, where it dissociates rapidly into two $\mathrm{O}$ atoms. One of these atoms stays with the Co while the other migrates to a $\mathrm{Pd}$ site. The reasons for the mechanism are not yet clear, but the bimetallic mixture apparently combines the bond-breaking abilities of Co with the high electrochemical potential of $\mathrm{Pd}$. A trimetallic mixture containing $\mathrm{Pd}, \mathrm{Co}$, and Mo proved to be especially effective, surpassing Pt by a small margin in its ability to reduce oxygen catalytically.

"Several catalysts have been found that rival $\mathrm{Pt}$," Bard said. In the future, this could lead to the ability to "make catalysts much cheaper than Pt."

Hans-Joachim Freund of the Fritz Haber Institute of the Max Planck Society in Berlin, Germany, wants to develop tools to bridge the gap between real catalysis, which takes place in various atmospheres and over a wide range of temperatures, and surface science, much of which is done in ultrahigh-vacuum conditions. In his plenary lecture, "Oxide Supported Nanoparticles: Models in Catalysis at Atomic 
Resolution," he expressed a desire to "capture the complexity of the real thing."

Freund has been studying $\mathrm{Au}$ on $\mathrm{MgO}$ substrates. Although $\mathrm{Au}$ is unreactive in bulk form, it becomes extremely reactive in nanoparticle form, capable of oxidizing $\mathrm{CO}$ below room temperature. He and his colleagues theorized that this effect was probably due to interaction between $\mathrm{Au}$ and the substrate. To investigate this possibility, the researchers deposited Au epitaxially on an $\mathrm{MgO}$ substrate and studied single $\mathrm{Au}$ atoms on the peaks, edges, and steps of the $\mathrm{MgO}$ surface using a custommade scanning tunneling microscope operating at $4 \mathrm{~K}$.

"The defects in oxides do all the chemistry," Freund said. As such, it is desirable to be able to engineer surface defects. Specifically, Freund would like to control the formation of F centers, or color centers, that occur when an oxygen atom is removed from the $\mathrm{MgO}$ lattice. These defects can contain two, one, or no electrons, and are thought to be important in $\mathrm{Au}$ catalysis: when a $\mathrm{Au}$ atom sits on one of these sites, its charge changes.

Using an electron spin resonance (ESR) spectroscopy device of their own design, with ultrahigh vacuum and infrared detection included, the researchers were able to learn a lot about the interaction of $\mathrm{Au}$ with the substrate surface. The free $6 \mathrm{~s}$ electron in Au takes on a more $p$-like character and less $s$ character when located in a color center defect, for example. Furthermore, when $\mathrm{CO}$ is introduced to the catalyst surface, ESR can differentiate $\mathrm{CO}$ located on a $\mathrm{MgO}$ terrace from $\mathrm{CO}$ on a cluster of $\mathrm{Au}$ atoms, and from $\mathrm{CO}$ sitting on a single $\mathrm{Au}$ atom. Interestingly, $\mathrm{CO}$ becomes slightly positive and Au slightly negative. Changing the thickness of the $\mathrm{MgO}$ layer can change the charge of $\mathrm{Au}$ atoms. "Even simple reactions are strongly influenced by the presence of a substrate," Freund concluded.

"At the nanoscale, interfaces are everything," stated Roy Clarke of the University of Michigan Applied Physics Program, USA, at the beginning of his plenary lecture, "Epitaxial Interfaces: A New World of Nanomaterials." The ability to image buried interfaces at the atomic scale is the key to understanding them and developing better devices. The ultimate goal is to be able to image the ultrafast movements of atoms at the interfaces-essentially creating a movie of atomic motion.

Clarke, whose research involves use of the Advanced Photon Source at Argonne National Laboratory, is convinced that the new third generation of storage rings for the production of $\mathrm{x}$-ray synchrotron radiation is a major step toward this ultimate goal. This new generation includes a device called an undulator, which causes electrons in the ring to wiggle back and forth, emitting $x$-rays with each wiggle. The result is a partially coherent, quasimonochromatic x-ray beam with a brilliance that exceeds that of the previous generation by many orders of magnitude.

Using this $x$-ray source and a technique called coherent Bragg rod analysis (COBRA), researchers were able to image nine monolayers of $\mathrm{GaSb}$ grown on InAs at atomic resolution. Clarke believes that the future fourth-generation x-ray synchrotron source will produce picosecond $\mathrm{x}$-rays that will enable researchers to make atomic movies.

George R. Newkome of the Departments of Polymer Science and Chemistry at the University of Akron, USA, gave a plenary lecture on "Nanoarchitectures by Self-Assembly Processes," in which he demonstrated the capabilities of a material called "terpy-Ru-terpy" to self assemble into hexameric rings and other structures. "Terpy" is short for "terpyridine." A pyridine molecule is a benzene ring with a nitrogen atom substituted for one of its carbon atoms. Terpyridine consists of three pyridine molecules bonded together to form a three-sided half-shell. Inserting a ruthenium atom between two of these terpyride molecules causes them to bond together to form a ring with $\mathrm{Ru}$ in the center. This "terpy-Ru-terpy" structure selfassembles under the right conditions to form a large, stable hexameric ring structure in which $\mathrm{Ru}$ does not migrate.

The hexamer can take in 12 electrons and give back 12 electrons, suggesting its possible use as a battery. By substituting other metals such as Fe or Os for some of the $\mathrm{Ru}$ atoms in the hexamer, researchers can change the electrical properties of the structure. The opening in the center of the hexamer can be enlarged by adding long organic substituent molecules as side chains on the terpyridine; Newkome said that by putting a single-walled carbon nanotube through the center of such a hexamer, one might be able to build a molecular motor. The hexamer is known to bond to the external walls of carbon nanotubes, and with the right metal substitution, can bridge the gap between the ends of two nanotubes, essentially polymerizing them. The possibility of connecting large numbers of carbon nanotubes together to form long chains in this way may be of interest to researchers trying to make nanotube or nanorod wires. The fact that the hexamer is also a fractal suggests that other fractal materials may be made using this chemical process.

Fractals are self-similar objects on mul- tiple scales, meaning that a small portion of a fractal will resemble the whole object. "Fractals have a 'fractional dimension,' as opposed to the integer dimension of regular geometric objects," explained Alexander Balankin of the Instituto Politécnico Nacional, Mexico, in his plenary lecture, "Fractal Concepts in Material Sciences." Common examples of fractals are the shapes of tree branches and the coastlines of countries.

Over the years, Balankin and his colleagues have used fractal analysis to explain the structure of cracks in concrete, the growth of sol-gels, and the scattering of microwaves and radar from soil. He described these investigations along with work on rough interfaces in disordered media, which focused largely on paper: torn paper, burned paper, and ink-stained paper. In each case, fractal structures emerge from ordinary phenomena.

\section{Nanostructured Materials and Nanotechnology}

Presenters in the symposium on Nanostructured Materials and Nanotechnology focused on the synthesis, processing, characterization, and modeling of nanomaterials and nanocomposites. F. Gordanenijad (Univ. of Nevada, Reno, USA) gave a brief review of the principles of magnetorheological (MR) fluids, along with a description of the most novel applications of these materials. Gordanenijad presented a unified method to predict the pressure drop of a MR fluid flow by using only the knowledge of physical properties of MR fluid, wall surface properties, and the applied magnetic field, without any use of the concept of yield stress, concluding that shear yield stress is not a material property for MR fluids.

D.G. Rickerby (European Commission's DG Joint Research Centre, Inst. for Environment and Sustainability, Italy) explained how nanostructured gas sensors based on nanocrystalline $\mathrm{SnO}_{2}$ thin films are able to detect concentrations of carbon monoxide at ppm levels, and nitrogen dioxide at $10^{-1}$ pmm levels. The gas sensing mechanism is based on measuring the change in conductivity due to chemisorption of gas molecules at the surface of a thin metal oxide film. In these sensors, both sensitivity and selectivity are temperature-dependent and can be increased by doping with a thin layer of platinum. Rickerby concluded that solid-state sensors are a viable alternative to traditional air-quality monitoring systems, making it possible to gather accurate real-time environmental information and with increased spatial resolution.

N.E. Walsöe de Reca (CINSO-CONICET- 
CITEFA, Argentina) presented a study on the electrical properties of nanostructured, heavily yttria- or samaria-doped ceria ceramics as a function of grain size by the use of electrochemical impedance spectroscopy. She reported a remarkable enhancement in the total ionic conductivity in nanostructured samples as compared with the intrinsic bulk conductivity of these materials. This effect was attributed to the predominance of grain-boundary conduction in the nanostructured materials, coupled with an increase in the grain-boundary ionic diffusivity with decreasing grain size.

M. José-Yacamán (Univ. of Texas at Austin, USA) in his invited talk presented a study of the structure of bimetallic Au-Pd nanoparticles by the use of molecular dynamics simulations and electron microscopy measurements. Yacamán showed that the shape of the nanoparticles is dictated by the kinetics of the growth process, and that the final shape of the particles is characterized by a rough surface and specific sites for $\mathrm{Pd}$ atoms surrounded by gold, which is likely to be linked to the well-known high catalytic activity of this kind of particles.

C. Kisielowski (Lawrence Berkeley Natl. Lab., USA) highlighted the relevance of the technological advancements in electron microscopy to study structure and chemical composition at the nanoscale. In particular, he remarked how information loss that comes from projecting the threedimensional materials structure into a two-dimensional image can be circumvented with new developments on instrumentation. He gave application examples of the most recent advancements on electron microscopy, such as the characterization of dislocations in GaAs:Be and investigations of strain relaxation processes in FePt nanoparticles.

R. Guirado-López (Universidad Autónoma de San Luis Potosí, Mexico) devoted his talk to showing how the combination of molecular modeling with several experimental techniques plays a fundamental role in the understanding of the properties of nanomaterials. He analyzed by electronic structure calculations the adsorption properties and gas storage capacity of different carbon nanostructures, finding, for example, that when small thiol molecules are adsorbed on a $\mathrm{C}_{60}$ molecule, unexpected adsorbed phases are obtained, characterized by the formation of molecular islands at the surface of the $\mathrm{C}_{60}$.

During the poster session, M.A. Gracia Pinilla (Universidad Autónoma de Nuevo León, Mexico) presented an experimental and theoretical work on the formation and

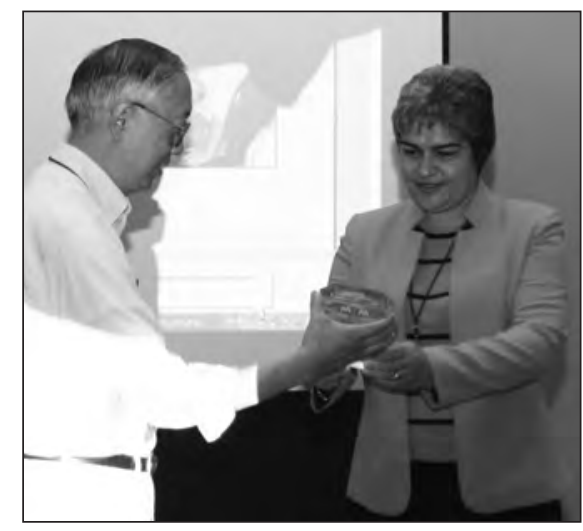

María Guadalupe Chacón Monárrez (right), secretary of education and culture for the state of Chihuahua, Mexico, receives a plaque from R.P.H. Chang of Northwestern University, honoring her leadership. Chacón Monárrez introduced the Materials World Modules (MWM) program in her region. MWM is developed under Chang's direction.

structure of tetrahedral Ag nanoparticles. Gracia explained the synthesis process of Ag tetrahedra by magnetron sputtering and their characterization with x-ray diffraction, scanning electron microscopy, transmission electron microscopy, atomic force microscopy, scanning Auger microscopy, and UV-visible spectroscopy. The obtained nanoparticles were about 5 $\mathrm{nm}$ in size, with a deviation of less than $2 \%$. The stabilization of the geometry of the particles was verified through the use of molecular dynamics simulations, and using density functional theory (DFT) calculations, Gracia Pinilla showed that this kind of structure is stable even at smaller sizes. Gracia discussed some of the main characteristics of the surface of the particles and the potential relevance of these features on catalytic processes.

\section{Theory and Computer Simulation of Materials}

The symposium Theory and Computer Simulation of Materials emphasized computational techniques and theoretical developments regarding the structural, electronic, magnetic, optical, and superconducting properties of materials. Invited speaker A. Michaelides (Fritz Haber Inst. of the Max Planck Society, Germany) discussed his development of DFT models based on materials such as water, ice, and salt. Presenting scanning tunneling microscope images of water clustering on $\mathrm{Cu}(111)$ surfaces, Michaelides said that the models looked like a "molecular waltz" as the hydrogen and oxygen molecules moved around each other. In his discussion of salt, Michaelides said that the surface energy for salt is between $10 \mathrm{meV}$ and $15 \mathrm{meV}$ and that he discovered that, unexpectedly, chlorine atoms on a clean sodium chloride surface tend to adhere to the chlorine rather than the sodium atoms.

M. Springborg (Univ. of Saarland, Germany) presented a theoretical treatment of chain compounds and nanoscale materials. Based on a study on chains of various metals inside a mordenite host material, Springborg showed that if the distance between the lead atoms in the host can be controlled, a semiconductor wire can be formed. By changing the distance between the atoms the bandgap of that semiconductor can be modified, he said. Gold chains were particularly interesting, as an increase in the bond length finally led to the gold chain splitting into pairs of gold dimers in which one of the pairs had small bond length spacing between the atoms and the other had a larger bond length.

\section{Photovoltaics, Solar Energy Materials, and Thin Films}

The symposium on Photovoltaics, Solar Energy Materials, and Thin Films provided an overview of the leading thin-film PV technologies and new developments in polymer photovoltaics, materials characterization, and transparent top-cell materials for tandem devices.

N.G. Dhere (Florida Solar Energy Center, USA) provided an update on $\mathrm{CuInGaSe}_{2}$ (CIGS) commercialization technologies and key challenges in research and development to meet the projected gigawatts of $\mathrm{CuInSe}_{2}$ (CIS) module production. C. Ferekides (Univ. of South Florida, USA) discussed the major challenges in CdTe-based PV technology and pointed out two major research areas: CdTe film treatments and metal contacts.

Innovative approaches in the development of a wide-bandgap material for tandem applications were presented from the group of A.D. Compaan (Univ. of Toledo, USA). The incorporation of a small amount of $\mathrm{Mg}$ opened the bandgap of CdTe significantly, paving the way for the development of a CdMgTe/CdS top cell that transmits more than $60 \%$ of the radiation with energy less than the bandgap of CdMgTe. X. Mathew (CIEUNAM) discussed a processing procedure that gives better stability for the $\mathrm{CdMgTe}$ film during standard $\mathrm{CdCl}_{2}$ vapor treatments.

S. Quinones (Univ. of Texas at El Paso, 
USA) demonstrated smooth CdTe film growth on CdTe(111) substrates using a conventional close-spaced sublimation (CSS) reactor. Work at this laboratory is focused on improving the surface of these films and demonstrating epitaxial growth on $\mathrm{CdTe}(111)$ and $\mathrm{CdTe}(211)$ substrates for both solar cell and infrared detector applications.

The potential of soft $x$-ray spectroscopy in revealing the electronic and chemical properties of interfaces in thin-film solar cells was discussed by C. Heske (Univ. of Nevada, Las Vegas, USA). Focusing primarily on the $\mathrm{CdS} / \mathrm{Cu}(\mathrm{In}, \mathrm{Ga})(\mathrm{S}, \mathrm{Se})_{2}$ interface, Heske demonstrated how chemical interdiffusion processes influence and, in this particular system, even optimize the electronic band alignment at the interface. During the discussion period, Heske showed some of the first results using synchrotron-based $x$-ray emission spectroscopy to elucidate $\mathrm{Cu}$ and $\mathrm{CdCl}_{2}$ treatments in CdTe thin-film solar cells.

M. Reyes-Reyes (Universidad Autónoma de San Luis Potosí, Mexico) described a fabrication procedure that enhanced the efficiency of an organic solar cell from $2.5 \%$ to $5.2 \%$. The 1-(3-methoxycarbonyl)propyl-1-phenyl- $(6,6) \mathrm{C}_{61}$ (PCBM) elongated nanostructures can assemble spontaneously within an organic host through annealing to form high-efficiency devices. These PCBM elongated nanostructures form under optimal conditions involving thermal gradients that provide fluidic mobility for the fullerenes, allowing greater dispersion of nanocrystalline PCBM within regioregular poly(3-hexylthiophene) (P3HT).

\section{Archaeology and Arts Issues in Materials Science}

In the symposium on Archaeology and Arts Issues in Materials Science, researchers discussed methods used in the characterization, consolidation, and conservation of prehistoric, archaeological, and art materials such as metals, stone, ceramic, wood, textile, and bone, along with other objects such as mural paintings and codex. Invited speaker R. Nieto Hernández (Universidad Autónoma del Estado de México) described archaeology as an essential component for obtaining knowledge of the ancient world and society. He showed masks from ancient sites in Mexico. He also showed x-ray fluorescence data from a number of pottery samples and related these to modifications of production methods and movements of the population.

L.A. Torres Montes (UNAM) introduced his topic as "a problem everyone knows and no one speaks about":

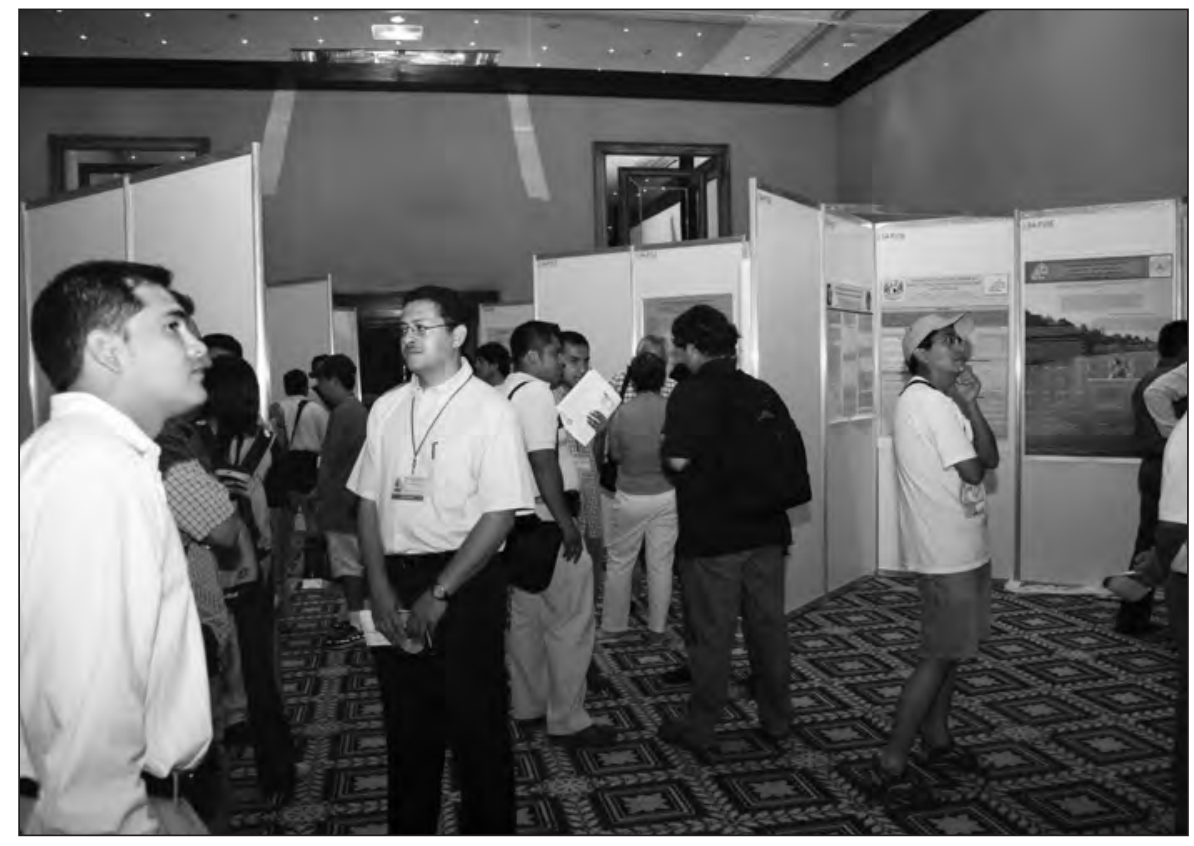

Attendees discuss materials research with colleagues at the poster session during IMRC 2006.

Cleaning procedures have inadvertently opened the door to an unfortunate invasion in architectural marbles. Marble is often cleaned abrasively or chemically, and recent practice has favored oxalic acid as an environmentally friendly method with far less material removal than abrasion. Regrettably, historic marbles have developed dark stains after cleaning. Torres Montes definitively identified oxalic acid as the culprit. Lichens produce oxalic acid themselves to extract ions from stone. To have a ready-made reservoir of oxalic acid in a marble is too good for the opportunistic lichens to resist. Torres Montes has found more secure ways to clean the stone and to cap it with a sealing layer.

\section{Materials Characterization}

The symposium on Materials Characterization demonstrated the need for advanced tools to address difficult materials research problems. E. Bedolla (Universidad Michoacana, Mexico) opened the symposium with a discussion of the physical characterization and interfacial and capillary phenomena in liquid processing of metal matrix composites (MMCs). He said that the interface between reinforcements and the matrix is undoubtedly one of the most important features in a composite system since it affects the mechanical properties of the materials through mechanisms such as debonding, stress damping, crack devia- tion, and grain-boundary pinning. He has worked for several years on MMCs using $\mathrm{TiC}, \mathrm{AlN}, \mathrm{Al}_{2} \mathrm{O}_{3}$, and $\mathrm{SiC}$ as the reinforcement and $\mathrm{Al}$ alloys, $\mathrm{Mg}$ alloys, and $\mathrm{Cu}$ alloys as the matrix.

J. García López (Centro Nacional de Aceleradores, Spain) described his work on using transmission electron microscopy and non-Rutherford backscattering to measure structural damage produced by ion implantation in $\mathrm{SiC}$ single crystals. A dose of $10^{14}-10^{15}$ ions $/ \mathrm{cm}^{2}$ displaces $1 \%$ of the atoms, he said. In studying the carbon sublattice, García López was able to measure the progress of the damage by changing the energy of the incident beam during the measurement.

T. Kryshtab (Instituto Politécnico Nacional-ESFM, Mexico) spoke about the problem of microstructural and texture characterization of polycrystalline materials. While pole figures from neutron scattering provide large-scale grain orientation information deep in the material, and x-rays can do the same to a depth of several hundred micrometers, these techniques unfortunately fall short of providing microstructural information such as grain size distribution and defect density. The goal is to have a technique that is both nondestructive and volume-sensitive; surface techniques are always prone to sample surface preparation and, in general, cannot provide averages over grains as thoroughly as a three-dimensional technique. Kryshtab provided a solid theoreti- 
cal analysis for using extinction, a dynamical effect in Bragg scattering, to dig out additional insights.

A. Hurd (Los Alamos Natl. Lab., USA) presented a survey of the advantages of neutrons, focusing on problems in residual stress and materials deformation. Since neutrons have exceptional penetrating power, they lend themselves well to diffraction studies in composites wherein the amount of load carried by each phase can be determined independently. Strain mapping near a weld has also been a powerful neutron research activity, Hurd said. Other advantages of neutrons include sensitivity to light elements, isotopic labeling, and magnetic scattering, he said.

\section{Synchrotron Radiation and Neutron Sources in Materials Research}

During the past decade, continued depletion of energy resources and the effects on the global environment has been the concern of every industrial economy in the world. A long-term solution can only be achieved through new breakthroughs in energy harvesting, energy storage, energy conversion, energy transport, and environmental influence-all of which require development and understanding of energy materials and energy processes at molecular, nanoscopic, and mesoscopic scales. The major user facilities, including nanocenters, neutron sources, and synchrotron radiation sources, provide a unique set of tools to address energy materials problems. This was the major focus of the symposium on Synchrotron Radiation and Neutron Sources in Materials Research.

E. Isaacs (Argonne Natl. Lab., USA) opened the symposium with a discussion of the revolutionary new materials and material architectures required for harvesting solar energy or for reversible storage of hydrogen fuel. Isaacs gave several recent examples such as the semiconductor nanoparticle-DNA composites for potential photovoltaic applications in which the use of synchrotron radiation revealed the charge separation process. He also described how the unique synthetic organic scaffold structures revealed by the use of synchrotron radiation are responsible for biomimetic (Fe-hydrogenase) hydrogen production.

Another theme pursued at the symposium was the realization of new structures that are capable of hydrogen storage approaching the U.S. Department of Energy's target of $6 \mathrm{wt} \%$ by 2010 . This included a new class of metalorganic nanoporous frameworks with tunable pore size and functionality described by
T. Yildirim (Natl. Inst. of Standards and Technology, USA) and clathrate hydrates, described by K. Loshkin (Univ. of Tennessee, USA), both using neutron elastic and inelastic scattering. By using both x-ray photoelectron spectroscopy (XPS) and x-ray absorption spectroscopy (XAS), A. Nilsson (Stanford Synchrotron Radiation Lab., USA) showed that the hydrogenated carbon bonds are formed in carbon nanotubes, allowing hydrogen storage of nearly $5.1 \mathrm{wt} \%$. Understanding the chemical and physical interactions of hydrogen with material surfaces, interfaces, and in confined geometries is key to controlling the performance of catalysts, nanostructured materials, and membranes, which form integral parts of hydrogen-based fuel cells. Examples of using neutron sources was presented by J.Z. Larese (Univ. of Tennessee; Oak Ridge Natl. Lab., USA) to study molecular interaction in carbon nanotube arrays, and by K.W. Herwig (Oak Ridge Natl. Lab., USA) to investigate diffusion of hydrogenous species in membrane fuel cells.

An industrial perspective on developing the optimum compositions and processing conditions for real-world cathode materials in solid-oxide fuel cells was the topic of discussion by Y. Gao (GE Global Research), who has used high-energy x-ray powder diffraction methodology toward this goal with synchrotron radiation. D.-J. Liu (Argonne Natl. Lab., USA) presented an in situ investigation on the promise of aligned carbon nanotubes as a low-cost alternative for $\mathrm{Pt}$ electrodes in a protonexchange membrane fuel cell (PEMFC) using XAS. A similar approach by C. Segre (Illinois Inst. of Technology, USA) has elucidated the electronic states and local chemistry of the metal atoms in the nanoparticle catalysts PtRu in a direct methanol fuel cell (DMFC). In situ small-angle x-ray scattering (SAXS) and grazing incidence small-angle $x$-ray scattering (GISAXS) studies of a new class of materials developed by dispersing layered silicates with polymers at the nanoscale were discussed by G. Sandí (Argonne Natl. Lab., USA). These materials that are suitable for battery applications show a relaxation of polymer chains inside the clay layers above room temperature, allowing the Li ions to have very high conductivity.

\section{Ferroelectricity and Piezoelectricity}

Presentations focusing on the understanding of ferro- and piezoelectric materials utilizing theoretical, experimental, and/or novel characterization methods were an important theme in the symposium on Ferroelectricity and Piezo- electricity. M.W. Cole (Army Research Laboratory, USA) and S.P. Alpay (Univ. of Connecticut, USA) highlighted experimental work focused on achieving temperature stability for ferroelectric $\mathrm{Ba}_{1-x} \mathrm{Sr}_{x} \mathrm{TiO}_{3}$ (BST)-based tunable devices for communications applications. The attainment of temperature stability is not trivial, since the capacitance of BST-based devices is strongly influenced by ambient temperature changes. By creating a material design consisting of a systematically varied stoichiometric composition BST thin film with $\mathrm{Ba} / \mathrm{Sr}$ ratios ranging from $60 / 40$ to $90 / 10$ - referred to as a functionally graded/multilayer film composition-the researchers were able to control the magnitude and the sign of the temperature coefficient of capacitance (TCC) of the BSTbased thin-film material in a coplanar or vertical device configuration. Such a design, with smooth linear compositional gradients normal to the substrate, possesses internal stress and polarization gradients that can be tuned to improve with the temperature sensitivity of the materials performance. Cole and Alpay reported significant improvements in materials temperature stability whereby the $\mathrm{TCC}_{20-90}$ was lowered by $88 \%$ with respect to the uniform composition of $\mathrm{Ba}_{0.60} \mathrm{Sr}_{0.40} \mathrm{TiO}_{3}$. What is most impressive about this material design is that the improvements in lowering the material TCC were accompanied by improvements in both dielectric tunability and loss at microwave frequencies. Furthermore, preliminary device modeling results demonstrated that using such a simple functionally graded film in a varactor device configuration would yield a 0.8-db improvement in insertion loss at the $\mathrm{K}_{\alpha}$ band. The attainment of temperature-stable tunable devices eliminates the need for expensive device packaging, which allows enhanced performance at lower costs.

Alpay provided an overview of recent theoretical results focused on phasetransformation characteristics of the prototypical ferroelectric barium titanate grown epitaxially on cubic and orthorhombic substrates. The fundamental analysis, based on a thermodynamic approach, demonstrated that monoclinic and even triclinic phases may be realized in films that arise from point group symmetry reduction due to the mechanical boundary conditions imposed by the substrate. These results indicate the possibility of tailoring desired electrical and electromechanical properties by engineering the internal stress state. Such tailoring permits predictable, controllable, and reproducible device properties to be realized, which could enhance performance, 
reliability, and property-uniformity of electronic devices and sensors.

Since the feasibility of a future spintronics device using multiferroic $\mathrm{BiFeO}_{3}$ is still debated because of its leaky nature, weak ferroelectricity, and lack of understanding of origin of magnetism, R.S. Katiyar (Univ. of Puerto Rico) offered an alternative approach of increasing polarization and decreasing leakage current by cationic dop- ing with other elements. This work investigated the effect of doping on the ferroelectric and ferromagnetic properties whereby $\mathrm{La}$-doped $\mathrm{BiFeO}_{3}$ (La on Bi site) and La- and $\mathrm{Cr}$-doped $\mathrm{BiFeO}_{3}$ (La on $\mathrm{Bi}$ site and $\mathrm{Cr}$ on Fe site) in both polycrystalline and epitomical thin films were synthesized and evaluated for leakage characteristics and ferroelectric properties. La substitution enhanced the multiferroic properties of $\mathrm{BiFeO}_{3}$ and reduced the leakage current by several orders of magnitude. Further enhancement of the properties was observed by doping in both $\mathrm{Bi}$ and Fe sites with $\mathrm{La}$ and $\mathrm{Cr}$, respectively. The higher values of polarization and magnetization in epitaxial films compared with polycrystalline counterparts were explained on the basis of the structural studies and epitaxial constraints between the film and the substrate.

\section{Roundtable on Science Education Focuses on Global Cooperation}

During the welcoming session opening the conference, Eugenio Cetina Vadillo (Dirección General de Educación Superior Universitaria) addressed the serious challenges faced by the Mexican educational system when the number of students in higher education increased by a factor of 85 in recent decades, and how the system rose to meet these challenges. Specifically, the 10,000 scholarships awarded through the Faculty Enhancement Program ensured that faculty had the proper educational qualifications to teach the next generation of students. From 1997 through 2005, about 1000 academic staff positions were added per year throughout Mexico. As a result, Cetina Vadillo said, "Mexico's contribution to mainstream world science has more than doubled since 1994."

At the roundtable discussion on science education held midweek during the conference, R.P.H. Chang (Northwestern Univ., USA) discussed the role that can be played by materials science and engineering education to meet worldwide challenges in healthcare, environmental protection, and energy. These areas of global concern are linking countries and regions in new ways, said Chang. Nations are seeking improvements in science education that will enable them to train future generations of scientists and engineers as well as increase the overall science literacy of their citizens. In the United States, Chang has been promoting the Materials World Modules (MWM) program for use in middle school and high school education. MWM has produced a series of interdisciplinary modules based on topics in materials science, including composites, ceramics, concrete, biosensors, biodegradable materials, smart sensors, polymers, food packaging, and sports materials.

A year ago, María Guadalupe Chacón Monárrez (secretary of education and culture for the state of Chihuahua, Mexico) introduced the MWM program in her region. With 3.2 million people, Chihuahua makes up 3.2\% of the Mexican population and produces $4.4 \%$ of the gross domestic product. In a mission to improve advanced education enrollment in science and technology, the state trained high school teachers in implementing the modules in their classrooms. They began with the pilot program on the materials science of concrete, which was translated into Spanish.

Guillermo Fernández de la Garza, executive director of the United States-Mexico Foundation for Science (FUMEC), also said that the MWM program has been instrumental in providing focus in the classroom. Based on a National Science Resources Center program in the United States, FUMEC is teaching teachers methods to develop thinking skills using teaching units. For example, children are guided through an exploration of sugar, alum, talc, baking soda, and cornstarch as an exercise in observation. These hands-on modules could be developed further by MWM with the aim of creating posi- tive feedback at the secondary level, said Fernández de la Garza. Some of this effort is focused on teachers, and many resources are freely available online. The Exploratorium's Institute for Inquiry is one such resource; the Exploratorium is working toward an agreement with the science center in Mexico City for partnerships.

At the level of advanced education, J. Lever Huffmaster, director of development and cooperation for the CONACYT, Mexico, said that postgraduate degrees in Mexico total about 16,800 per year in sciences and related fields, excluding the arts. Mexico's master's students outnumber Brazil's, but the reverse is true for doctoral students. Even though the population with $\mathrm{PhD}$ degrees is small, the numbers have doubled in 10 years, Lever Huffmaster said. Lever Huffmaster said that there are not enough private-sector jobs to drive the PhD market yet. Mexico's plan is to provide incentives for postgraduate studies through fellowships to students and stipends to mentors. The government is also creating postdoctoral positions and supporting professors who are the most active in training PhD candidates.

Mexico's college-level programs are also being enhanced by international collaborations. The Universidad Politécnica de Madrid (UPM) in Spain, represented at the roundtable by José de Frutos Vaquerizo, has established cooperation arrangements with many Latin American universities and doctoral programs, including four in Mexico. These programs are executed by telecommuting and video conferencing. UPM has 3000 undergraduate students, $300 \mathrm{PhD}$ candidates, 200 master's students, and 190 Erasmus scholars (scholars selected for an intra-European student-faculty mentoring program). UPM supports the Institute of Solar Energy and the Institute for Systems Based on Optoelectronics and Microtechnology. The university is piloting an international system of accreditation in Europe as part of a sweeping educational reform movement that is to be in place by 2010.

During the roundtable discussion, Chang also introduced the Global School for Advanced Studies, a new program designed to prepare young researchers to collaborate effectively across sectors, regions, and disciplines and to address global issues that are beyond the capabilities of individual countries and regions.

The organizers of IMRC 2006 also did their part in promoting materials education by increasing the number of students participating in the technical program throughout the week. The Secretaría de Educatión Pública, Mexico, supported student attendance at the conference, and 113 graduate and undergraduate students were among the attendees of the 10 tutorial sessions that preceded the technical program. Many of the tutorials were presented in Spanish to accommodate student needs. 


\section{New Trends in Polymer Chemistry and Characterization}

The symposium on New Trends in Polymer Chemistry and Characterization covered a large variety of subjects. Among the outstanding and interesting findings reported throughout the symposium, some attracted particular attention for their originality.

For instance, F. Charra (Energie Atomique, France) discussed the parallel manipulation of individual molecules, which is an important goal in many current research projects in the nanosciences. This aim requires the confinement of molecular motion inside nanoscale cavities and channels in order to allow advanced filtering operations based on single-molecule characteristics as Charra discussed. Charra constructed two-dimensional molecules on carbon surfaces, where the bond lengths of the molecules matched the atomic spacing of the carbon atoms. $\mathrm{He}$ was able to form molecular filters with this structure. Compared with threedimensional molecular sieves such as zeolites, these structures are capable of being monitored using high-speed, low-current scanning tunneling microscopy. In addition, the whole active region is exposed to the environment to enable molecular manipulation, and the fabrication is based on self-assembly that can be modified to change the size of the cavities. Different structures with different pore sizes can be formed using pentacene, hexabenzocoronene, and coronene. For larger molecules, there is no absorption by the layer. Intermediate-sized molecules display adsorption and trapping, while small molecules show absorption and then surface diffusion.

N. Solladié (Laboratoire de Chimie de Coordination, France) talked about an adjustable cavity for host-guest recognition in cofacial bis-porphyrinic tweezers. She recently discovered that an electronic coupling was generated between the two porphyrins of such small tweezers when a molecule of pyrazine was inserted into the cavity, generating a 1/1 host/guest complex. The enhanced stability observed in the complexation of pyrazine by that dimer was ascribed to the preorganization of the $\mathrm{Zn}(\mathrm{II})$ bis-porphyrin.

Conjugated hyperbranched polymers are currently one of the most attractive research fields in polymer chemistry. B.Z. Tang (Hong Kong Univ. of Science \& Technology, China) reported the synthesis and light-emitting properties of hyperbranched poly(aroylarylene)s and their use as precursors to magnetic ceramics. This work was presented by M. Häußler (Hong Kong Univ. of Science \& Technology, China). He talked about the synthesis of novel conjugated hyperbranched polyarylenes, polyyens, and poly(ferrocenylenesiyne)s by polycyclotrimerization and polycoupling of alkynes. These polymers exhibit a variety of functional properties including low intrinsic viscosities, high thermal stabilities, high light-emitting efficiencies, superb optical-limiting performance, and high light refractivity. To enrich this research field, Häußler reported the synthesis of a group of new soluble, conjugated, hyperbranched polymers containing fluorene, carbazole, triphenylamine, and ferrocene moieties by polycyclotrimerizations of bis(aryl ethynyl ketone)s.

B. Wandelt (Technical Univ. of Lodz, Poland) spoke about polymeric sensory systems based on molecular imprinting technology for processing and monitoring. Molecular imprinting of polymers (MIPs) provides a powerful approach to controlling electronic and optical properties of materials and obtaining working sensory systems. In Wandelt's work, molecular imprinting was realized by copolymerization of functional monomers in the presence of a target molecule that acts as a molecular template. Subsequent removal of the template left behind functionalized cavities that are able to recognize the template molecule. The size and shape of the cavity as well as the electronic structure inside the cavity are able to accept the template. The important element of the cavity is a receptorone of the functional monomers. The interactions between the template and the receptor inside the cavity changes the properties, which can be transduced outside the material as an electrical and/or optical signal. Wandelt and co-workers developed the MIP sensor, in which one of the functional monomers serving as the receptor is fluorescent; the fluorescence of the polymer is changeable depending on the analyte binding. The MIPs were polymerized as bulk porous materials as well as thin films. Wandelt developed a methodology for surface molecular imprinting of polymers (SMIPs) for the identification of peptides and larger biological objects such as cells. This technology leads to surface-imprinted cavities with molecular memory of a particular molecular system template. The template, by means of a biological object, forms a complex supramolecular structure with functional monomers during the preparation and polymerization processes.

\section{Surface Engineering and Thin Films}

The goal of the Surface Engineering and Thin Films symposium was to work toward achieving a full understanding of the nanoengineering process that will enable optimization of materials for device applications and enhance commercialization prospects. Laser shock processing is a technique for strengthening metals that can be used on finished products. G. Gómez-Rosas (Centro Universitario de los Lagos, Mexico) described his experiments using a Nd:YAG laser at $1064 \mathrm{~nm}$ to deliver 8-ns pulses of $2.5 \mathrm{~J} / \mathrm{cm}^{2}$ to a sample of Ti-6Al-4V. The impact of the pulses produced a shock wave in the material, which induced a compressive residual stress field; a hole-drilling method was used to confirm the presence of this stress field. Gómez-Rosas said that the residual stress strengthens metals by increasing fatigue crack initiation time, reducing the fatigue crack growth rate, and increasing wear resistance.

J.F. Chavez Lara (Instituto Tecnológico de Chihuahua, Mexico) presented results of a study of the electrical conductivity of a polymer called poly(ethylene naphthalene2,6-dicarboxylate), or PEN, at high electric fields. By performing dielectric analysis on two samples, one amorphous and one semicrystalline, under a vacuum of $10^{-4} \mathrm{~Pa}$ at room temperature, Chavez Lara and his colleagues recorded a sharp break in current versus voltage plots; this break is the threshold energy, which marks the start of increased current flow in the material. Specifically, current density increased with increasing sample crystallinity.

In order to elucidate the chemical mechanisms for the formation of thin films of titanium, Y. Cruz Martínez and her coworkers at the Instituto Tecnológico de Morelia, Mexico, started with a novel metalorganic precursor called tetradiethylenamine titanium (TDEAT), which has the formula $\mathrm{Ti}\left[\mathrm{N}\left(\mathrm{CH}_{2}-\mathrm{CH}_{3}\right)_{2}\right]_{4}$. Starting with a simulation of the reaction of TDEAT with $\mathrm{N}_{2}$ and $\mathrm{O}_{2}$ using modeling software called FACT, the researchers proposed a mechanism and then performed experiments to try to verify it. The model involves calculation of the free energy for a wide range of possible reactions, elimination of reactions with a positive free energy change, and further reduction of the remaining reactions using Hess's law. Experimentally, the researchers made Ti thin films with a very homogeneous morphology using metalorganic chemical vapor deposition, and analyzed the resulting films and residual gas using $\mathrm{x}$-ray photoelectron spectroscopy (XPS) and Fourier transform infrared spectroscopy. XPS showed that the films were composed of $\mathrm{Ti}, \mathrm{O}, \mathrm{N}$, and C. Reaction products in the gas phase included $\mathrm{CO}, \mathrm{CN}, \mathrm{NH}_{3}, \mathrm{OH}, \mathrm{CH}_{3}$, and various hydrocarbons. The experimental results closely matched the proposed 
chemical mechanism obtained from the simulation.

In an attempt to synthesize monolayers that have a structurally active transition when exposed to an electrical charge, R. Terrill (San Jose State Univ., USA) and his co-workers have synthesized organomercaptan monolayers of 1,1-mercaptodecanoic acid and 3-mercaptopropionic acid on gold surfaces. They propose that in such monolayers, the application of a negative charge to the Au surface will cause the organomercaptans to stand up straight, effectively acting as an open valve to allow flow through pores in the surface. Conversely, application of a positive charge should cause the organomercaptans to lay down on the surface, effectively closing the pores. The researchers succeeded in producing dilute monolayers of both compounds and will perform tests of their NEM properties in the near future.

\section{Novel Building Materials and Technologies}

Among the highlights of the symposium on Novel Building Materials and Technologies was a report on the synthesis and performance of a photoluminescent ceramic material and a photoluminescent inorganic binder obtained at room temperature. Inorganic amorphous polymers were used as a matrix for these photoluminescent crystals. Optical and microstructural properties were performed, and a stable photoluminescent inorganic system was obtained. Another highlight was the presentation of a computer simulation of a pressure-volume-temperature (PVT) process as a coupled thermomechanical nonlinear dynamic problem, which was in good agreement with PVT experiments.

The application of smart materials in the construction industry is an emerging technology. The aim of one of the studies presented was to determine the piezoresistive behavior of cement-based composites subjected to shear loading. It was observed that lower load speeds produced a higher shear strength. Furthermore, in the elastic zone, a direct relationship was observed between mechanical load and electrical resistance; in the plastic zone, an inverse relationship was observed.

Expansive soils are a problem for the construction industry internationally, since they produce structural damage due to volume variations caused by humidity. Soil treated with lime has resulted in volumetric soil stabilization. A study was presented on the influence of the humidity variation on the final mixture strength.

A modeling approach for fiberreinforced concrete, based on simulating the rigid phase with finite elements ran- domly distributed within the concrete matrix, was presented. Results showed that an elastoplastic model is a good approach for studying behavior under loads.

\section{Fracture Mechanics}

Processing, structure, and properties of metals and alloys, ceramics, and composites were addressed in the Fracture Mechanics symposium. By applying Fourier spectroscopy techniques to laminated materials, E. Andrés-Zárate (Universidad Juárez Autónoma de Tabasco, Mexico) and his associates demonstrated the ability to identify and measure small cracks in materials that may not be detectable by other means. When the diffraction patterns obtained from illumination of the surface of alumina samples with a He:Ne laser were analyzed using Fourier transform methods, the resulting spectrograms revealed the rectangular, circular, or triangular shape of small fractures. These patterns changed when the materials were subjected to mechanical or thermal stresses. This technique could be used to study the formation dynamics of small cracks in laminar materials both qualitatively and quantitatively.

\section{Nanoscience at the Intersection of Physics and Biology}

The symposium on Nanoscience at the Intersection of Physics and Biology showed that progress in the development of theoretical first-principles methods makes reliable nanostructure simulations in physics and biology feasible.

In the section on clusters, J. Jellinek (Argonne Natl. Lab., USA), S.N. Khanna (Virginia Commonwealth Univ., USA), and I.L. Garzón (Universidad Nacional Autónoma de México) presented theoretical insight into the electron structure of these systems. These presentations were complemented by a section about cluster spectroscopy by K.H. Bowen (the Johns Hopkins Univ., USA), D.G. Leopold (Univ. of Minnesota, USA), M.B. Knickelbein (Argonne Natl. Lab., USA), and G. Ganteför (Univ. of Konstanz, Germany).

The current frontier of DFT developments and applications to nanoscale systems was presented in three sections by D.R. Salahub (Univ. of Calgary, Canada), A. Köster (CINVESTAV, Mexico), A. Reber (Virginia Commonwealth Univ., USA), A. Vela (CINVESTAV, Mexico), M. Castro (Universidad Nacional Autónoma de México), C. Quintanar (Universidad Nacional Autónoma de México), A. Goursot (CNRS, France), and L.L. Boyer (Naval Research Lab., USA).

Two sections with contributions from S.B. Trickey (Univ. of Florida, USA), K. Jug
(Univ. of Hannover, Germany), H. Chacham (Universidade Federal de Minas Gerais, Brazil), A. Bansil (Northeastern Univ., USA), G. Geudtner (CINVESTAV, Mexico), F. Janetzko (CINVESTAV, Mexico), S. Hirata (Univ. of Florida, USA), and S.L. Richardson (Howard Univ., USA) were devoted to bulk materials.

Approaches for the study of biomaterials were presented in two sections by M.R. Pederson (Naval Research Lab., USA.), A.A. Quong (Thomas Jefferson Univ., USA), L. Noodleman (Scripps Research Inst., USA), H.A. Duarte (Universidade Federal de Minas Gerais, Brazil), and J.M. Sullivan (Virginia Commonwealth Univ., USA).

\section{Ecomaterials}

In the symposium on Ecomaterials, a diverse range of results were presentedfrom theory to applied technological development-on environmentally friendly materials science. Exploiting agriculture and agricultural waste was a strong theme. Experimental materials derived from processed wood fiber abound, taking advantage of a major natural resource in South American countries.

The subject of sustainability is especially reaching a critical crossroads. The paradigm of materials research in this area needs to change, as evident from the discussion that took place among the speakers and audience. Environmental sustainability refers to the long-term maintenance of valued environmental resources in an evolving human context. The best way to define and measure sustainability is under debate. Economists often emphasize an accounting approach that focuses on the maintenance of capital stocks. Some in the environmental realm focus instead on natural resource depletion and on whether current rates of resource use can be sustained into the distant future.

\section{Optical Characterization of Materials}

In his opening presentation for the symposium on Optical Characterization of Materials, D.E. Aspnes (North Carolina State Univ., USA) said that metrology is going from thin films to three-dimensional structures. Using spectroscopic ellipsometry, Aspnes recorded signals from structures that he then compared with a model. With this technique, Aspnes was able to achieve $0.1 \%$ accuracy. He said that the technique enables researchers to determine the refractive index, the dielectric constant, and the thickness of the structure simultaneously.

A. Whitley (HORIBA Jobin Yvon, Inc., 
USA) described the use of hyperspectral (spectroscopy with microscopy) imaging to increase the speed of Raman spectroscopy. The system couples atomic force microscopy (AFM) and surfaceenhanced Raman scattering (SERS) and measures near-field Raman signals. The AFM tip is coated with one of the SERSactive metals such as gold or silver. The AFM moves the coated tip close to the sample surface, which then produces a surface-enhanced Raman signal around the tip. The signal is detected using farfield optics, and every pixel in the image produced by scanning across the surface has the full spectrum. Whitley said that the device can be used in three ways: point mapping, line scanning, and global imaging. The third technique defocuses the system into a circle which degrades the spectrum and is not used much by the Raman community. An inverted microscope is used to illuminate the sample from below. The system produces 200-nm resolution over sampling of 5-10 times, and $50-\mathrm{nm}$ resolution is thought to be a future reality.

\section{Non-Molecular Solids}

Far from the classical and somewhat obsolete distinction between inorganic and organic materials, non-molecular solids are the basis of many interesting applications of solids in materials science.
Invited speaker R. Sáez Puche (Universidad Complutense de Madrid, Spain) covered the history of pigments of various colors. He showed that zeolites of the sodalite-type framework, wherein four sodium atoms form a cage, could be used to produce a synthetic blue ultramarine. This material is very cost-effective in the coloration of paints and plastics due to its chemical and thermal stability and low toxicity. It replaces many of the heavy elements that are currently used as pigments. The pigment is being manufactured commercially.

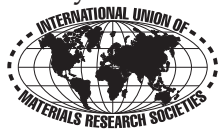

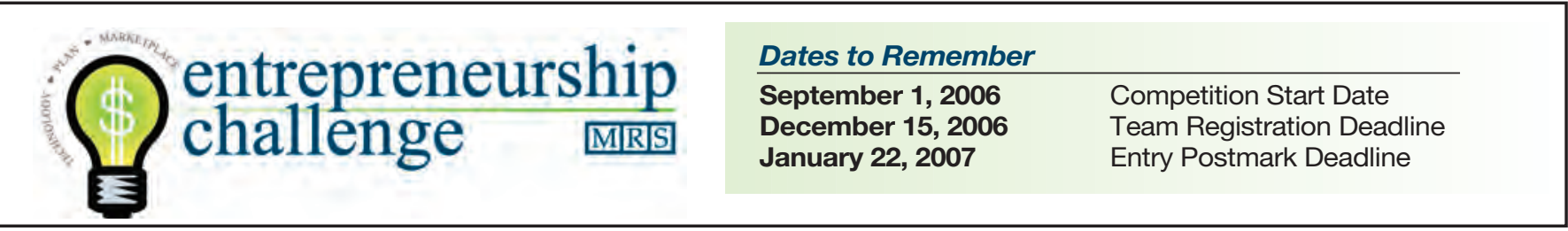

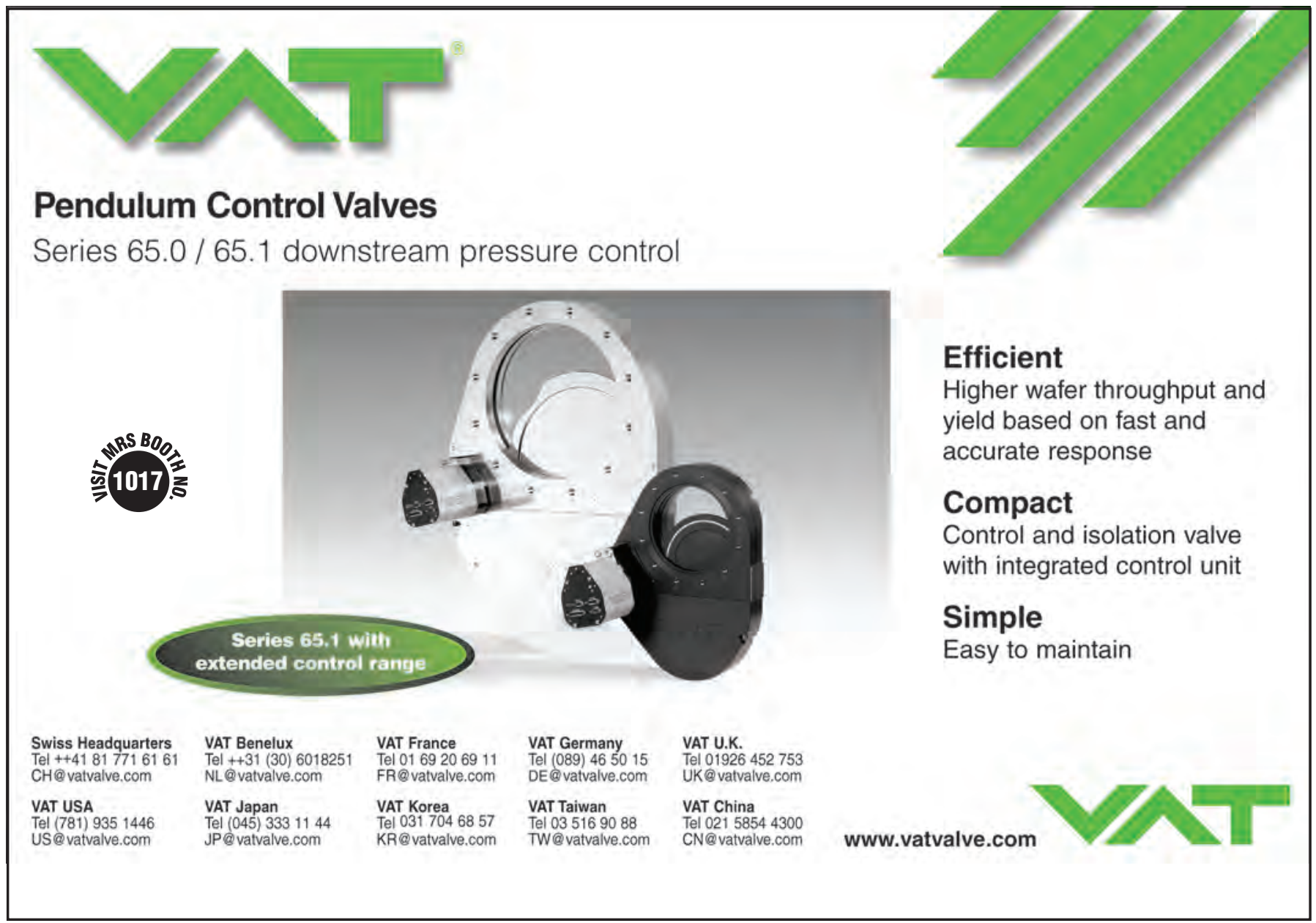

For more information, see http://www.mrs.org/bulletin_ads 